\title{
Violencia, delito y trastorno psíquico
}

\section{Néstor Ricardo Stingo ${ }^{1}$, Mónica Santamaría ${ }^{2}$, Liliana Noemí Avigo ${ }^{3}$, María Cristina Zazzi ${ }^{4}$, Carlos Luis Gatti ${ }^{5}$, Guillermo Martínez Perez ${ }^{6}$}

\section{Introducción}

Paradójicamente, junto con el aumento de la expectativa de vida, se produce un aumento de las situaciones en que ésta se ve comprometida a partir de la violencia.

La violencia promueve, actualmente, mayor volumen de muerte que las enfermedades cardiovasculares y crea circunstancias de desestabilización que afectan tanto al medio social como a la trama psíquica de cada uno de los sujetos.

La violencia es un fenómeno arcaico; una multitud de pensadores, incluidos los historiadores de la civilización, se han preocupado por el análisis de los factores que concurren para fomentarla, condenando los estragos que derivan de su incontrolada incidencia tanto en el nivel individual como en el de la vida institucional.

El hombre es un animal dotado de sin razón, al decir de E. Morin, que ha alcanzado atisbos de racionalidad y en el que pesan filogenéticamente los niveles anteriores. La racionalidad es una de las conquistas de la evolución biológica y no siempre parece gobernar las acciones. Si bien la violencia es un fenómeno transtemporal, está sujeta, en la pluralidad de sus formas, a factores históricos cambiantes. La reflexión sobre su esencia y sus consecuencias en la vida privada y en el campo social, se ha multiplicado en nuestro tiempo. En los medios de comunicación más diversos aparece claro un aumento en la extensión, frecuencia e intensidad de la violencia, unida a la comprobación del temor referido a la inermidad de los diferentes sectores de la comunidad frente a ella.

\footnotetext{
${ }^{1}$ Jefe del Departamento de Docencia e Investigación en el Hospital Psicoasistencial Interdisciplinario José T. Borda.

${ }^{2}$ Médica Psiquiatra. Ayudante de la Cátedra de Salud Mental. Facultad de Medicina. U.B.A.

${ }^{3}$ Jefa del Servicio de Atención Primaria I en el Hospital Psicoasistencial Interdisciplinario José T. Borda.

${ }^{4}$ Jefa de Sección del Servicio de Atención Primaria I en el Hospital Psicoasistencial Interdisciplinario José T. Borda.

${ }^{5}$ Psicólogo de Planta del Servicio de Atención Primaria I en el Hospital Psicoasistencial Interdisciplinario José T. Borda.

${ }^{6}$ Médico Forense de la Justicia Nacional.
} 
El avance de las ciencias neuropsíquicas, a partir del conocimiento de la bioquímica cerebral, neurorreceptores y neurotransmisores; y de los mecanismos intrapsíquicos, ha permitido una mayor comprensión de las conductas de los seres humanos y de los conflictos vinculares. La pérdida de valores e identificaciones de tipo estructurante crea un desequilibrio que se manifiesta en trastornos psíquicos con gran impacto social, como son, en especial, los trastornos antisociales de la personalidad y el abuso de sustancias psicoactivas.

Una de las preguntas acuciantes de nuestro tiempo es, si la ciencia en general y la psiquiatría en particular, pueden aportar elementos a los fines de dilucidar y limitar este fenómeno de inusitada extensión e intensidad en nuestros días.

El planteo dominante en esta investigación consiste en cuantificar, en una población determinada, el trastorno psíquico existente y su posible relación con los delitos cometidos.

\section{Recursos conceptuales}

La complejidad del fenómeno y la heterogeneidad de sus manifestaciones requieren la utilización de recursos conceptuales que permitan organizar y delimitar el aparente desorden en que se presentan los hechos.

La palabra agresividad, tiene su origen en la raíz latina aggredi, que significa avanzar, acercarse, atacar, en el sentido de tocar, que luego desemboca en el acto de comprender. Sólo modernamente, agresión significa ataque latente ${ }^{7}$. Cada uno puede darle a la agresión un sentido diferente y simbolizarla de una manera particular. Cuando la agresión se transforma en destructiva, estamos en presencia de lo que denominamos violencia. La violencia que se apoya en la exacerbación de los mecanismos neurobiológicos de la respuesta agresiva, tiene un carácter destructivo sobre las personas y/o los objetos y supone una profunda disfunción social.

Por su parte, hostilidad (agresividad anormal), tiene su raíz en la palabra latina "hostilis" que significa enemigo o contrincante.

Violencia viene de "violare", violar una norma o ley. Incluye toda relación de tipo destructivo para sí o para terceros, contando con el medio físico como objeto de ataques posibles.

La violencia forma parte de diversos contextos. Puede generarse en forma espontánea o facilitada por diferentes circunstancias, como el abuso de tóxicos, alcohol y drogas

\footnotetext{
${ }^{7}$ Stingo N., Zazzi C., Gatti C. Violencia e Institución. Revista Emergencias. Publicación Periódica del Servicio de Emergencia I del Hospital José T. Borda. 1991.
} 
y los "ismos" políticos y religiosos. Puede darse, como reacción inesperada,

intempestiva, que parece responder a resortes inconscientes escapando al control del sujeto. También puede obedecer a actos reflexivos. Es una dimensión desintegrante.

\section{Hipótesis sobre la naturaleza de la violencia}

Es conveniente distinguir entre causas y condiciones de las causas de la conducta violenta, estableciendo entre ellas correlaciones y probabilidades. Cada aspecto de una condición depende de otros, por lo tanto son siempre elementos relativos.

Siendo la violencia un fenómeno estrechamente ligado a la condición de lo psíquico encontramos, al querer explicarla, una multiplicidad de determinantes. El hombre es una amalgama de naturaleza y cultura y está forzado a vivir simultáneamente en

ambos mundos, que no sólo lo rodean sino que lo penetran hasta su más profunda intimidad, habrá que buscar las respuestas en las direcciones naturalistas y culturalistas que se disputan la preferencia en todo intento de explicación.

Diversas interpretaciones se han elaborado en esas direcciones, sobre la naturaleza de la violencia.

Freud, que desarrolló la teoría de las pulsiones, en la última etapa, equiparó agresión con pulsión de muerte.

E. Fromm afirmó que hay dos tipos de agresividad humana. Una es la instintiva, biológicamente adaptativa, puesta al servicio de la supervivencia del individuo y de la especie, con asiento neurofisiológico en ciertas áreas del cerebro, sobre todo en el sistema límbico. La otra es caracterial, puesta en marcha cada vez que el hombre se siente amenazado en sus valores, símbolos e instituciones. ${ }^{8}$

Ningún ser humano puede vivir mucho tiempo sin ser reconocido; dicho reconocimiento, así como la satisfacción de necesidades elementales, puede buscarse en ocasiones recurriendo a la violencia.

La teoría de la agresión debida a la frustración llega a una conclusión errónea: sin frustración no habría agresión; lo cual instaura un prejuicio hacia la agresión. Se debe conceder a la frustración una ubicación importante en nuestra comprensión de la violencia, pero no es el único factor involucrado en ella. ${ }^{9}$

\footnotetext{
${ }^{8}$ Vidal G., Bleichmar H. y Usandivaras R. Enciclopedia de Psiquiatría. Editorial El Ateneo. Buenos Aires. Segunda Edición. 1979.

${ }^{9}$ Gunn John. Violencia en la Sociedad Humana. Editorial Psique. Buenos Aires. 1976.
} 
Diversos grados de destructividad pueden correlacionarse con diferencias en las estructuras sociales. El potencial destructivo aumenta a medida que se incrementan los avances de la tecnología ${ }^{10}$.

Los grandes desarrollos y sus efectos sobre el mundo social modifican la estructura personal y hacen que aparezcan fenómenos antes inadvertidos o bien nuevos en el surgimiento y dinámica de la violencia y de las personalidades que la ejercen. Toda violencia debe ser interpretada dado que es expresión de algo.

Desde hace 150 años, muere una persona cada sesenta segundos. En los últimos 50 años, se triplicó la expectativa de vida, pero se redujo el lapso entre muertes de 60 a 20 segundos ${ }^{11}$. En EE UU, uno de cada 4 homicidios ocurre dentro de una relación de parentesco entre víctima y victimario ${ }^{12}$.

La violencia se incuba con frecuencia en el seno de la familia y se refuerza en la sociedad, se ha vuelto casi consuetudinaria y hasta anodina. La accesibilidad a la violencia es la característica de nuestra época. La presencia de acontecimientos violentos en la televisión, el cine y otras manifestaciones culturales, produce un gran impacto por la intensa fascinación que siempre ejercieron estos actos en los seres humanos. Los niños norteamericanos ven, entre los 5 y 15 años de edad, la aniquilación de un promedio de 13.400 personas $^{13}$.

En la televisión norteamericana, los programas infantiles muestran actos de violencia extrema cada 16,3 minutos promedio, sin contar con los dibujos animados que producen verdaderas orgías de crueldad.

Los niños que presentan preferencia por los programas violentos de televisión, son más agresivos que los que no la tienen (Eron - 1963), y esta relación se mantiene en distintos grupos sociales. Hay experimentos de laboratorio y de campo que confirman que ver violencia puede tener efectos causales en la agresividad de los niños ${ }^{14}$.

La violencia proyectada como imagen, permite al espectador, al igual que la ficción, identificarse con lo que ve. La imagen invade la sensibilidad y el ámbito de lo espectacular, trivializando así la violencia al transformarla en algo cotidiano. La mediatización despoja a la violencia de su sentido histórico y su contexto. La ficción y la

\footnotetext{
${ }^{10}$ Fromm Erich. Anatomía de la Destructividad Humana. Editorial Siglo XXI. Sexta Edición. Madrid. España. 1980

${ }^{11}$ Hacker F. Agresión. Editorial Grijalbo S.A. 1973

${ }^{12}$ Echeburúa E. La Delincuencia Juvenil: Factores Predictivos. Cuaderno del Instituto Vasco de Criminología I. $35-49$

${ }^{13}$ Hacker F. Agresión. Editorial Grijalbo S.A. 1973.

${ }^{14}$ Delgado Bueno S., Psiquiatría Legal y Forense. Volumen I. Pg 543.
} 
realidad se mezclan en las mentes. La información, en cambio, restituye a la violencia al contexto que le ha dado origen y mueve a la crítica.

Para Ostermeyer, la sociedad crea estados que hacen agresivos a los hombres, y esa agresividad se descarga en la misma sociedad, la que propicia los canales necesarios para su drenaje. Otra parte se reprime y se manifiesta como autoagresión, tóxicodependencias, suicidio, enfermedades y neurosis.

Desde hace 10 años se observa un incremento impresionante de las manifestaciones violentas en las ciudades del mundo desarrollado. Sus principales protagonistas son, en general, individuos cada vez más jóvenes de las zonas suburbanas marginales. La tensión racial y la discriminación, marginación y exclusión social por distintos motivos, avivan el fuego de la violencia.

Distintos equipos de investigadores sociales norteamericanos especializados en génesis y fenomenología de la violencia, coinciden en la gravitación que tiene el surgimiento de grupos humanos discriminados frente al acontecer de la violencia social.

La transformación producida por los medios de comunicación de masas, el calor destacado de los bienes materiales, la inmediatez de la información, la gran difusión y progresión de recursos de todo tipo, han producido cambios en las formas de violencia aunque el efecto de la misma sea semejante a épocas pretéritas.

Desde la perspectiva biológica se considera al organismo humano como un sistema abierto y autorregulable que a través de la evolución filogenética se ha caracterizado por la autonomía, la jerarquización de funciones y el predominio de la actividad del sistema nervioso central ${ }^{15}$.

Entre las primeras investigacones científicas de este siglo se encuentran las que Kluber y Bucy hicieron en 1939, observaciones de las conductas de monos rhesus a los cuales se les habían extirpado ambos lóbulos temporales. Estos animales se volvieron inusualmente mansos, sin signos de miedo ni agresividad, con hipersexualidad y exploración oral excesiva. En lo que respecta a la conducta agresiva se acepta que la mansedumbre inducida por las lesiones descriptas es el resultado de la supresión de las influencias de la amígdala. Por el contrario, la estimulación de la amígdala se acompaña con frecuencia de agresión ${ }^{16}$.

Hay otras regiones del SNC cuya lesión o estimulación modifican la conducta agresiva en algunas especies. Por ejemplo extirpación de la corteza cerebral en gatos y perros hacen que respondan ferozmente a estímulos mínimos. Efectos similares se observan en

\footnotetext{
${ }^{15}$ Geertz C., El Desarrollo de la Cultura y la Evolución de la Mente. La Interpretación de la Cultura. Cap III. Ed. Gedisa. 1990. Barcelona. España.

${ }^{16}$ Delgado Bueno S., Psiquiatría Legal y Forense. Volumen I. Pg 543.
} 
la lesión del séptum y núcleo accumbens en roedores.

La investigación de la localización neuroanatómica precisa del origen de la conducta violenta continua en nuestros días, en lo que respecta a los seres humanos vivos, a través de las nuevas técnicas de diagnósticos por imágenes como los mapeos cerebrales, tomografías, resonancia magnética y spect. Estos recursos radiológicos, que van aumentando rápidamente su capacidad de resolución, demuestran diferencias de la actividad cerebral en distintas áreas de la corteza de sujetos violentos con respecto a individuos control.

A medida que se avanzó en la investigación de la bioquímica del cerebro, se fue dando relevancia al papel de los neurotransmisores y los neuromoduladores en la regulación de la conducta agresiva. Diversos tipos de esta conducta tienen un sustrato distinto desde el punto de vista neuroanatómico y neuroquímico ${ }^{17}$.

Los estudios se han efectuado principalmente sobre noradrenalina y serotonina. A la primera se la relaciona con la agresión irritativa, ya que la estimulación de la amígdala provoca ira y cólera y disminuye la noradrenalina central. Diversos estudios dan cuenta de una relación inversa entre las tasas de serotonina en líquido cefalorraquídeo y la conducta agresiva; igualmente se halló una correlación inversa entre las puntuaciones de la Escala MMPI y las tasas de serotonina en el líquido cefalorraquídeo. En otros estudios se hallaron tasas más bajas de serotonina en Líquido cefalorraquídeo en los sujetos cuyos actos violentos eran impulsivos. Freud, en su carta a Einstein, reflejaba un sentimiento pesimista de la vida relacionado con la supresión de la agresión. Le decía: "No es probable que nosotros seamos capaces de suprimir las tendencias agresivas de la humanidad" ${ }^{\prime 18}$.

En realidad, el problema no es suprimir la agresividad que tiene que ver con la supervivencia biológica, sino controlar o limitar la conducta agresiva patológica o violenta. Para tal fin es fundamental el diagnóstico y evaluación de las personas violentas o potencialmente violentas, teniendo en cuenta que una conducta violenta en nuestra sociedad no necesariamente es síntoma de un trastorno mental.

En algunos trastornos mentales es más frecuente que aparezcan conductas violentas, como en los retrasos mentales, en las intoxicaciones provocadas por sustancias psicoactivas, en las lesiones cerebrales (demencias degenerativas, traumáticas, vasculares, epilepsias), y en los trastornos de la personalidad, entre otros.

Para la comprensión del fenómeno violento es necesario considerar al sujeto, su posible

\footnotetext{
${ }^{17}$ Vallejo Ruiloba J. De Flores T. Introducción a la Psicopatología y la Psiquiatría. III Edición. Editorial Salvat. Buenos Aires. 1991.
}

${ }^{18}$ Freud S. Carta a A. Einstein. Obras Completas. Editorial Biblioteca Nueva. III Edición. Madrid. España. 
patología, sus móviles, sus metas y si este hecho forma parte de su vida habitual o es tan sólo circunstancial.

Es imprescindible tener en cuenta, además del sujeto y su posible patología, la naturaleza de la acción, los medios utilizados, la significación de lo violentado, el contexto social y las características de la acción en cuanto a tiempo, lugar, modo y ocasión.

Los factores biogenéticos y los psicosociales no pueden separarse en cuanto a su influencia en la causalidad de la conducta violenta. La agresividad es, pues, tanto innata como adquirida y las situaciones por las que atraviesa en su vida familiar y social el individuo pueden regularla, canalizarla o exaltarla.

\section{Objetivos}

En el presente trabajo el objetivo principal es cuantificar, en una muestra de una población perteneciente a una Unidad Psiquiátrica Carcelaria, los trastornos psíquicos que éstos presentan y el tipo de delitos violentos cometidos

Tal cuantificación apunta a la búsqueda de la existencia de algún tipo de relación entre trastorno psíquico y tipo de delito.

\section{Material y métodos}

Se presenta una muestra representativa de casos tomados de un Servicio Psiquiátrico Carcelario de Varones, en los cuales existió una conducta delictiva caracterizada por agresividad y/o violencia dirigida a personas u objetos. Se ha estudiado a dichas personas desde el punto de vista clínico psiquiátrico y se las ha evaluado a través de entrevistas y consulta de la Historia Clínica correspondiente.

Se realizó una anamnesis con datos sobre sus antecedentes personales, heredofamiliares y examen psíquico del estado basal actual, para poder arribar a un diagnóstico sobre la presencia o no de trastorno psíquico. Las situaciones sociales, laborales y culturales, también estuvieron contempladas.

Los diagnósticos se basaron en el DSM IV (Manual Diagnóstico y Estadístico de los Desórdenes Mentales), de la Asociación Americana de Psiquiatría ${ }^{19}$.

La serie de sesenta y ocho casos estudiada se tomó al azar con pacientes internados en este Servicio durante el lapso de un año comprendido entre mayo de 1997 y mayo de 1998. 


\section{Análisis de los casos estudiados}

Se han tenido en cuenta las siguientes variables para confeccionar un protocolo exclusivo para esta investigación. Los resultados fueron volcados dentro de una base de datos confeccionada especialmente para el estudio de las conductas delictivas agresivas y/o violentas, cuyos ítems se transcribe:

I- Identificación.

II- Datos Personales (edad, escolaridad, lugar de nacimiento, lugar de residencia actual, estado civil, número de convivencias anteriores, religión, conversiones religiosas, estabilidad laboral, ocupación, nivel socioeconómico, con quién y dónde vive)

III- Antecedentes (de consumo de sustancias psicoactivas y motivo aparente del consumo inicial, familiares, personales, judiciales, médicos).

IV-Sobre el hecho (motivación, tipo de

delito). V- Síntesis del Psiquismo

(diagnóstico).

\section{Parámetros de expresión de la violencia (resultados)}

En los cuadros siguientes se toman 68 casos de acuerdo a la descripción enunciada anteriormente.

\section{Discusión}

En relación a la muestra escogida, debemos señalar que la misma fue obtenida con sujetos varones internados en un Servicio Psiquiátrico Carcelario de la Capital Federal de la República Argentina. Dichos sujetos incurrieron en conductas delictivas, agresivas y/o violentas y presentaron algún trastorno psíquico que requirió tratamiento fuera de la Unidad Carcelaria común.

Los casos estudiados se tomaron al azar en el lapso que transcurrió desde mayo de 1997 a mayo de 1998. 


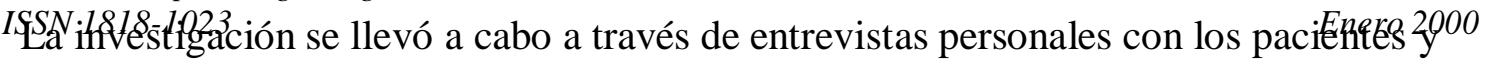
los datos consignados en las Historias Clínicas.

Las edades observadas marcan la mayor densidad en el grupo de 21 a 25 años (20 casos), seguido del grupo de 31 a 35 años (14 casos). La franja más densa es la que va de 21 a 35 años, que suma 42 casos.

Nuestra muestra marca un predominio de nacidos en la Capital Federal (30 casos).

Extranjeros: (6 casos). El Gran Buenos Aires y la Provincia de Buenos Aires cuentan con 20 casos.

En cuanto a la nacionalidad, 56 son argentinos y 6 extranjeros. En seis casos no consta el dato. El estado civil marca un franco predominio de los solteros (58.82\%), seguido de los casados, concubinos, viudos, separados y divorciados, en ese orden.

En relación a la estabilidad laboral, $23.53 \%$ la posee, $32.35 \%$ tenían un trabajo inestable y el

$44.12 \%$ no trabajaba.

La ocupación muestra un predominio de desocupados. 26.47\% fueron empleados y en otras actividades se repartió en no más de 6 casos cada una (profesionales, comerciantes, obreros). La escolaridad hallada demostró predominio de estudios primarios completos (32.35\%), seguidos de secundario incompleto (29.41\%). Se destaca que el 11.76\% (8 casos) era analfabeto.

El nivel socioeconómico demostró que un $47.5 \%$ eran de un estrato bajo.

En cuanto al consumo de drogas, el 70.59\% (48 casos) lo presentaba. Politoxicómanos en el $54.16 \%$, siguiéndole el $25.00 \%$ con consumo de marihuana y cocaína.

Al investigar el consumo de alcohol en nuestra casuística mostró que el 47.6\% consumía bebidas alcohólicas con habitualidad, siendo el consumo mixto de diversas bebidas el más frecuente (16 casos).

Los diagnósticos fueron efectuados siguiendo los criterios del DSM IV. A tal efecto cabe consignar la diferencia entre Rasgos y Trastornos de la Personalidad, siendo los primeros aspectos prominentes de la misma que no implican patología, definiendo la personalidad como "patrones de conducta profundamente entramados que incluyen el modo en que uno se relaciona, percibe y piensa sobre el entorno y sí mismo". Los trastornos de la personalidad suponen patrones rígidos y desadaptativos, de una gravedad suficiente como para causar un déficit en la capacidad adaptativa o un sentimiento subjetivo de malestar. Nuestra casuística demostró un $73.53 \%$ de trastornos de la personalidad (50 casos), siguiéndole las psicosis en un $14.7 \%$. 


\section{Conclusiones}

Nuestras conclusiones fundamentales son las siguientes:

- E1 73.53\% de los casos estudiados fueron trastornos de la personalidad.

- El tipo de delito más frecuentemente cometido fue el robo o el hurto (58.82\%), seguido de las lesiones (leves y graves $-17.65 \%$ ) y el homicidio en el $11.76 \%$.

- El consumo de drogas (en este casos interpretadas como sustancias psicoactivas diferentes del alcohol) estuvo presente en el $70.59 \%$ de los casos.

- El consumo de alcohol demostró presencia efectiva en el $47.6 \%$ de los casos.

- El nivel socioeconómico era bajo en el $47.05 \%$ de los casos. El 44.12\%, eran desocupados. El presente estudio nos ha permitido observar que la mayoría de los pacientes internados en esta Institución no presentaban una estructura psicótica. Por el contrario, fueron diagnosticados como trastornos de la personalidad con predominio de rasgos antisociales.

El estudio de los delitos cometidos permite inferir que los mismos pertenecen en su mayoría al robo-hurto, delito contra la propiedad y con el cual se buscaría algo considerado como rédito. No se trató, en la mayoría de los casos, de injurias contra las personas en las cuales podríamos pensar en un descontrol o en una actuación impulsiva violenta. De acuerdo a los datos obtenidos debemos destacar el peso de las circunstancias sociales intervinientes como posibles facilitadores de las conductas delictivas violentas a través de la desocupación y el uso de drogas.

El panorama que presenta la violencia en nuestros días requiere la participación integradora, tanto de las ciencias neuropsiquiátricas como de las sociales a los fines de abordar esta problemática.

El presente trabajo aporta observaciones que podrían clarificar puntos específicos de la problemática psicosocial de la violencia. 


\section{Bibliografía}

1.- Alonso Fernández F. Fundamentos de la Psiquiatría Actual. Editorial Paz Montalvo. Barcelona. 1979.

2.- Brown G.L., Ebert M.H., Goyer P.F. et al. Aggression in Humans Correlates eith Cerebrospinal Fluid Anime Metabolites. Psychiatry Res. 1, 131-

$139,1979$.

3.- Chrousos G. Regulation and Dysregulation of the Hypothalamic - Pituitary - Adrenal Axis. ("The Corticotropin releasing Hormone perspective"). Endoc. and Metab. Clin. N.A. Vol 21, N 4, Dec. 1992.

4.- D.S.M. IV. Criterios Diagnósticos. Editorial Masson. 1995.

5.- Dahl A. Heredity in Personality Disordes: An Overview. Clin. Genet. Vol 46. Spce. N Pg. 138. 1994.

6.- Delgado Bueno S. Psiquiatría Legal y Forense. Volumen I. Pg 543. Editorial Colex. Madrid. 1994.

7.- Dubovsky SL., Thomas M. Beyond Specificity Effects of Serotonin and Serotonergic Treatments on Psychobiological Dysfunction. J. Psychosom

Res. May 1995. 39(4). 429-44.

8.- Echeburúa E. La Delincuencia Juvenil: Factores Predictivos. Cuaderno del Instituto Vasco de Criminología I. 35-49.

9.- Ey H., et al. Tratado de Psiquiatría. Editorial Toray Masson. Barcelona. 1978.

10.- Freud S. Carta a A. Einstein. Obras Completas. Editorial Biblioteca Nueva. III Edición. Madrid. España.

11.- Freud S. Las Pulsiones Destinos Pulsionales. Editorial Biblioteca Nueva. III Edición. Madrid. España.

12.- Fridman N. El Conocimiento del Propio Ser Biológico. En: El Inconciente y la Ciencia. Amorrortu Editores. Buenos Aires. 1993.

13.- Fromm Erich. Anatomía de la Destructividad Humana. Editorial Siglo XXI. Sexta Edición. Madrid. España. 1980.

14.- Geertz C. El Desarrollo de la Cultura y la Evolución de la Mente. La Interpretación de la Cultura. Cap III. Ed. Gedisa. 1990. Barcelona. España. 
16.- Hacker F. Agresión. Editorial Grijalbo S.A. 1973.

17.- López Rey M. Criminología. Editorial Biblioteca Aguilar. Madrid 1975. Pg. 92.

18.- Lundberg U., Rasch B., et al. Physiological Reactivity and Type A Behavioer in Preschool - Maccagno A., y col. Estratategia Médico Legal ante una Catástrofe Colectiva.

El Caso A.M.I.A. Impresora J.R. s.r.l. Abril de 1996.

19.- Mas M. Correlatos Biológicos de la Violencia en Psiquiatría Legal y Forense.

Delgado Bueno y Colaboradores. Editorial Codex. Madrid. 1994. Pg. 1245.

20.- May Rollo. Fuentes de la Violencia. Emece Editores. 1974.

21.- Morin Edgard. El Paradigma Perdido. Editorial Kairós. Barcelona. 1992.

22.- Ricón L. Crisis de Fin de Siglo y Subjetividad. Revista Argentina de Psiquiatría Vertex. Suplemento I. Vol III - Primer Semestre 1997.

23.- Rivarela José María. Reflexión sobre la Violencia. Editorial Arandura. 1993.

24.- Staner L., Mendlewicz J. Biological Psychiatry and Current Clasification of Depressive Disorders. Encephale. Vol 17. N 3. Pg 179. 1991.

25.- Stingo N., Zazzi C., Gatti C. Violencia e Institución. Revista Emergencias. Publicación Periódica del Servicio de Emergencia I del Hospital José

T. Borda. 1991.

26.- Vallejo Ruiloba J. De Flores T. Introducción a la Psicopatología y la Psiquiatría. III Edición. Editorial Salvat. Buenos Aires. 1991.

27.- Vidal G., Bleichmar H. y Usandivaras R. Enciclopedia de Psiquiatría. Editorial El Ateneo. Buenos Aires. Segunda Edición. 1979. 
Cuadro 1

\section{ANEXOS}

\section{Edades}

\begin{tabular}{|l|r|r|}
\hline & Número & Porcentaje \\
\hline$D e 18$ a 20 años & 2 & 2.94 \\
\hline$D$ e 21 a 25 años & 20 & 29.41 \\
\hline De 26 a 30 años & 8 & 11.76 \\
\hline De 31 a 35 años & 14 & 20.59 \\
\hline De 36 a 40 añss & 4 & 5.89 \\
\hline De 41 a 50 años & 10 & 14.70 \\
\hline+ de 51 años & 10 & 14.70 \\
\hline Totales & 68 & $100 \%$ \\
\hline
\end{tabular}

Cuadro 2

\section{Escolaridad}

\begin{tabular}{|l|r|r|}
\hline & Nümero & Porcentaje \\
\hline Analfabeto & 8 & 11.76 \\
\hline Primario incompleto & 10 & 14.70 \\
\hline Primario Completo & 22 & 32.35 \\
\hline Securdario incompleto & 20 & 29.41 \\
\hline Secunctario completo & 4 & 55.88 \\
\hline Terciario incompleto &.- & 0.00 \\
\hline Terciario completo & 2 & 2.94 \\
\hline Unversitaro incompleto & $\ldots$ & 0.00 \\
\hline Universitario completo & 2 & 2.94 \\
\hline Totales & 68 & $100 \%$ \\
\hline
\end{tabular}




\section{Lugar de nacimiento}

\begin{tabular}{|l|r|r|}
\hline & Numero & Porcentuje \\
\hline Capitaf Federal & 30 & 4412 \\
\hline Gran Bs. As & 12 & 17.65 \\
\hline Provncia de Bs. As. & 8 & 11.76 \\
\hline Interior del pais & 6 & 8.82 \\
\hline Exteriot & 6 & 8.32 \\
\hline No consta & 6 & 8.82 \\
\hline Totales & 68 & $100 \%$ \\
\hline
\end{tabular}

Cuadro 4

Lugar de residencia

\begin{tabular}{|l|r|r|}
\hline & Numero & Porcentaje \\
\hline Capital Fedital & 42 & 61.76 \\
\hline Gran Bs. As & 14 & 20.59 \\
\hline Provincia de Bs. As & 4 & 5.88 \\
\hline Interior del pais & 4 & 5.88 \\
\hline Exterior & 0 & 000 \\
\hline No consta & 4 & 5.88 \\
\hline Totales & 68 & $100 \%$ \\
\hline
\end{tabular}

\section{Cuadro 5}

\section{Estado civil}

\begin{tabular}{|l|r|r|}
\hline & Numero & Porcentaje \\
\hline Soltero & 40 & 58.82 \\
\hline Casado & 10 & 14.7 \\
\hline Concubinato & 10 & 147 \\
\hline Separado & 2 & 2.94 \\
\hline Divorciado & 2 & 2.94 \\
\hline Viudo & 4 & 5.88 \\
\hline Totales & 68 & $100 \%$ \\
\hline
\end{tabular}


Matrimonios o convivencias anteriores

\begin{tabular}{|l|r|r|}
\hline & Nimero & Porcentaje \\
\hline No consta & 44 & 6470 \\
\hline Una & 16 & 23.53 \\
\hline Dos & 6 & 8.82 \\
\hline Mass de dos & 2 & 2.94 \\
\hline Totales & 68 & $100 \%$ \\
\hline
\end{tabular}

\section{Cuadro 7}

\section{Religión}

\begin{tabular}{|l|r|r|}
\hline & Numero & Porcentye \\
\hline No consta & 6 & 8.82 \\
\hline Ateo & 2 & 2.94 \\
\hline Católica & 50 & 73.53 \\
\hline Secta & 8 & 11.76 \\
\hline Otra & 2 & 2.94 \\
\hline Totales & 68 & $100 \%$ \\
\hline
\end{tabular}

En tres casos se observó conversión (de religión católica a secta)

\section{Cuadro 8}

\section{Estabilidad laboral}

\begin{tabular}{|l|r|r|}
\hline & Numero & Porcentaje \\
\hline Notrabaja & 30 & 4412 \\
\hline Trabajo inestable & 22 & 32.35 \\
\hline Trabajo estable & 16 & 23.53 \\
\hline Totsles & 68 & $100 \%$ \\
\hline
\end{tabular}




\section{Ocupación}

\begin{tabular}{|l|r|r|}
\hline & Numero & Porcentyje \\
\hline Desocupados & 30 & 4412 \\
\hline Obreros & 6 & 8.32 \\
\hline Enpleados & 18 & 26.47 \\
\hline Profesiond & 2 & 2.94 \\
\hline Comerciantes & 2 & 2.94 \\
\hline Orra & 10 & 14.70 \\
\hline Totales & 68 & $100 \%$ \\
\hline
\end{tabular}

Cuadro 10

\section{Nivel socioeconómico}

\begin{tabular}{|l|r|r|}
\hline & Número & Porcentaje \\
\hline No conta & 2 & 2.94 \\
\hline Indigente & 6 & 8.82 \\
\hline Bajo & 32 & 47.05 \\
\hline Medio & 20 & 29.41 \\
\hline A.jto & 8 & 11.76 \\
\hline Totales & 68 & $100 \%$ \\
\hline
\end{tabular}

\section{Cuadro 11}

\section{Vive con}

\begin{tabular}{|l|r|r|}
\hline & Numero & Porcentaje \\
\hline No contesta & 4 & 588 \\
\hline Solo & 18 & 26.47 \\
\hline Pactes & 16 & 23.53 \\
\hline Pareja & 6 & 8.82 \\
\hline Esposa e hijos & 18 & 26.47 \\
\hline Orros & 6 & 8.82 \\
\hline Totales & 68 & $100 \%$ \\
\hline
\end{tabular}


Cuadro 12

Dónde vive

\begin{tabular}{|l|r|r|}
\hline & Numero & Porcentaje \\
\hline No conta & 8 & 11.76 \\
\hline Villa & 10 & 14.70 \\
\hline Casa tomada & 2 & 2.94 \\
\hline Hotel & 6 & 8.82 \\
\hline Pensican o inquilinato & 12 & 17.65 \\
\hline Casa propa & 18 & 26.47 \\
\hline Casa alquilada & 8 & 11.76 \\
\hline Otro lugar & 4 & 5.88 \\
\hline Totales & 68 & $100 \%$ \\
\hline
\end{tabular}

\section{Cuadro 13}

\section{Consumo de drogas}

\begin{tabular}{|l|r|r|}
\hline & Numero & Porcentaje \\
\hline Presente & 48 & 70.59 \\
\hline Ausente & 20 & 29.41 \\
\hline Totiles & 68 & $100 \%$ \\
\hline
\end{tabular}

\section{Cuadro 14}

Tipo de drogas más frecuentemente consumidas en los 24 sujetos consumidores

\begin{tabular}{|l|r|r|}
\hline & Número & Potcentaje \\
\hline Cocana solamente & 6 & 12.50 \\
\hline Marihaana solamerde & 4 & 8.33 \\
\hline Marihuana + cocaina & 12 & 25.00 \\
\hline politoxicón anos & 26 & 54.16 \\
\hline Totales & 68 & $100 \%$ \\
\hline
\end{tabular}

\section{Cuadro 15}

\section{Consumo de alcohol}

\begin{tabular}{|l|r|r|}
\hline & Nútiero & Potcentrje \\
\hline Presente (*) & 32 & 47.06 \\
\hline Ausente & 36 & 52.94 \\
\hline Totales & 68 & $100 \%$ \\
\hline
\end{tabular}


(*) En estos 32 casos la bebida más consumida fue cerveza sola (10), vino solo (6), diversas bebidas (16)

\section{Cuadro 16}

Edad del inicio del consumo de sustancias psicoactivas

\begin{tabular}{|l|r|r|}
\hline & Número & Porcentaje \\
\hline No consta & 14 & 25.00 \\
\hline Entre 10 y 19 años & 28 & 5000 \\
\hline Entre 20 y 29 años & 10 & 17.86 \\
\hline Mas de 30 años & 4 & 7.14 \\
\hline Totsles & 56 & $100 \%$ \\
\hline
\end{tabular}

\section{Cuadro 17}

\section{Tipo de delito}

\begin{tabular}{|l|r|r|}
\hline & Numero & Porcentaje \\
\hline Leacones leves y graves & 12 & 17.65 \\
\hline H comicictos & 8 & 1176 \\
\hline Delitos sexuales & 2 & 2.94 \\
\hline Robo-hurto & 40 & 58.82 \\
\hline Otros delitos & 6 & 882 \\
\hline Totales & 68 & $100 \%$ \\
\hline
\end{tabular}

\section{Cuadro 18}

\section{Diagnósticos}

\begin{tabular}{|c|c|c|}
\hline & Numero & Porcentaje \\
\hline Preos: & 10 & 147 \\
\hline Debilidad mental & 6 & 882 \\
\hline E ralepaa & 2 & 2.94 \\
\hline $\begin{array}{l}\text { Trastornos de la } \\
\text { personalidad ( })\end{array}$ & so & 7353 \\
\hline Totales. & 68 & $100 \%$ \\
\hline
\end{tabular}

(*) Los trastornos de personalidad se subdividen en: antisociales (12), límite (2), mixtos (36). De los límite y mixtos, se hallaron rasgos antisociales en 22 casos. 


\section{() (-)}

Este texto está protegido por una licencia $\underline{\text { Creative Commons }} \underline{4.0}$.

Usted es libre para Compartir — copiar y redistribuir el material en cualquier medio o formato- y Adaptar el documento - remezclar, transformar y crear a partir del material - para cualquier propósito, incluso comercialmente, siempre que cumpla la condición de:

Atribución: Usted debe reconocer el crédito de una obra de manera adecuada, proporcionar un enlace a la licencia, e indicar si se han realizado cambios. Puede hacerlo en cualquier forma razonable, pero no de forma tal que sugiera que tiene el apoyo del licenciante o lo recibe por el uso que hace.

$\underline{\text { Resumendelicencia }}$ - Textocompletodelalicencia 